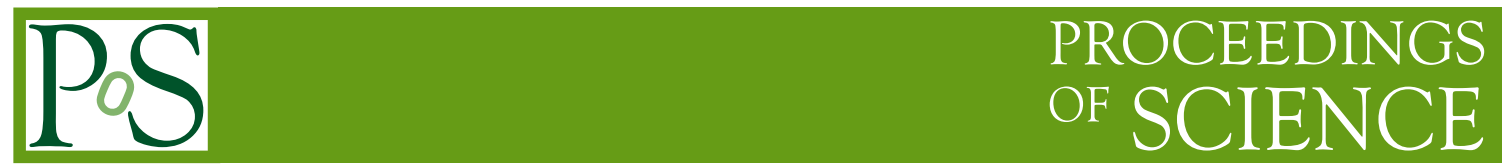

\title{
Nuclear Reactions And Neutrinos In Supernovae
}

\section{Silvio Cherubini*}

University of Catania and INFN-Laboratori Nazionali del Sud

E-mail: cherubinielns.infn.it

The interplay between Nuclear and Neutrino Physics is a major issue in Nuclear Astrophysics studies of explosive events, like Supernovae. Here we report on the intended studies in this field that will be carried on at INFN-Laboratori Nazionali del Sud, Catania, Italy, in the near future.

The 26th International Nuclear Physics Conference

11-16 September, 2016

Adelaide, Australia

${ }^{*}$ Speaker. 


\section{Introduction}

Nuclear Astrophysics studies got much interest in the last decades. As it is well known, one of the problems with these studies consists in the fact that cross sections between charged particle in the energy domain correspondent to astrophysical scenarios are generally very small (order of micro-, nano- picobarn and even less). This is due to the fact that the Coulomb barrier between the colliding nuclei is much higher than their relative energy.

In the beginning of the '90s of last century, the Nuclear Astrophysics group at INFN-Laboratori Nazionali del Sud (LNS) developed an efficient and reliable indirect method to measure such cross sections and hence overcoming some of the difficulties inherent in direct approaches. This method is called "Trojan Horse Method" (THM) after G. Baur [1]. With respect to the original Baur's idea, the method was actually implemented by considering the quasi-free reaction mechanism only and not a generic direct breakup one [2,3].

Slightly later than the Nuclear Astrophysics group (middle of the '90s of last century) a project for the construction of a large $\left(1 \mathrm{~km}^{3}\right)$ neutrino telescope that uses sea water as active volume was also launched at LNS. This project evolved into the KM3Net Collaboration [4] that involves 45 Institutions from 13 Countries. LNS are one of the leading Institutions in this project.

Research fields that are at the border between Nuclear Astrophysics and Neutrino Astrophysics can profit from this fortunate situation that emerged at LNS.

\section{Supernovae as Neutrino and Nuclear Physics laboratory}

Astrophysical events like Supernova (SN) explosions by their intrinsic nature require a large amount of interdisciplinary competences. Nuclear, particle and atomic physics knowledge is actually needed from the micro-physics side while the various phases of stellar evolution require expertise on cosmic and stellar models and on observational data. SNe can be regarded as a laboratory for fundamental physics and astrophysics, where neutrinos are key messengers to show any physical processes going on inside the exploding massive star. Therefore, neutrino signals and nucleosynthetic products induced by neutrino-nucleus interactions inside the $\mathrm{SNe}$ can be regarded as valuable observables in multimessenger Astronomy.

In principle, in order to really exploit the possible synergies mentioned above one should 1) study the capabilities of existing and future neutrino detectors and telescopes to observe low energy neutrinos originating from SN explosion or even from our Sun, 2) experimentally determine the cross sections of key nuclear processes involved in SNe nucleosynthesis with a particular emphasis on neutron induced reactions on short lived radioactive isotopes, 3) model the Pre-SN and SN phase together, as the pre-SN evolution of stars pinpoints the initial conditions of the SN explosion itself. It is well known (e.g. [5]) that massive stars evolve through stable nuclear burning stages until an iron core is formed. A key aspect of this evolution is that advanced nuclear burning are activated by few key reactions that release light particles (e.g., p, a, n) which then activate an enormous number of nuclear processes. Once the iron core is formed it becomes unstable inducing the explosion of the star and nucleosynthesis occurs within few seconds. For these reasons the study of the nucleosynthesis in both the pre-SN evolution and the explosion of massive stars constitute a powerful theoretical laboratory to use and test at the same time the values of the cross sections of nuclear 
reactions obtained from experiments.

Theoretical astrophysical predictions on SN neutrino flux spectrum can be tested by experimentally observing such spectrum using neutrino detectors and telescopes (see e.g. [4, 6]). The physics for neutrino-nucleus interactions is a basic ingredient in Monte Carlo generators used to simulate the response of neutrino detectors and telescopes to real events. At high energy, Relativistic Fermi Gas models [7] are often used with sufficient reliability but at very low energies, as in SN scenarios, this is not the case: e.g., effects of nuclear structure cannot be disregarded.

The neutrino-nucleus interactions important for the observation of the SN emitted neutrinos by neutrino detectors and telescopes are basically those with hydrogen and oxygen isotopes. While Hyper-Kamiokande is designed to detect low energy neutrinos, the observation of such neutrinos by the KM3Net telescope is still uncertain. In any case, assuming that KM3Net telescope will be able to detect low energy neutrinos, it will possibly be needed to take into account also the interaction of neutrinos with nuclei other than just hydrogen and oxigen, as it uses sea water as active volume. The basic theory for calculating the relevant cross sections for this application is well known (e.g. [21] ) and ancillary pieces of information can be derived from experimental data.

\section{Applying THM to SN studies: ${ }^{56} \mathrm{Ni}(\mathrm{n}, \mathrm{p}){ }^{56} \mathrm{Co}$}

THM proved to be a powerful tool not only for studying a number of reactions induced by stable nuclei but also by neutrons $[8,9,11,12,10,13,14,15,16,17,18]$.

As reported in the bibliographic references, the basic idea of THM is that a reaction of astro-

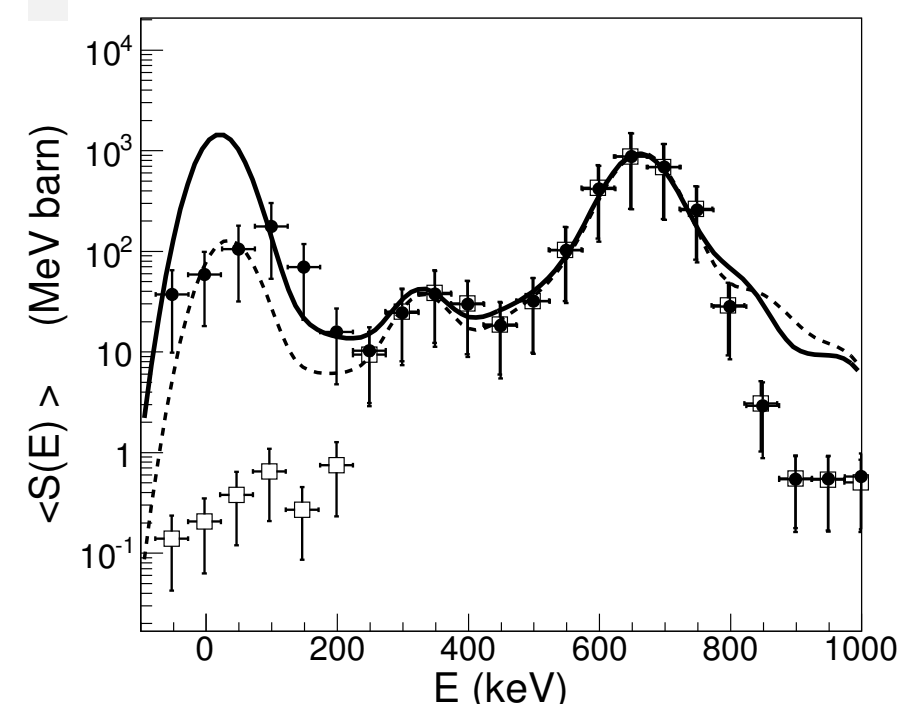

Figure 1: The ${ }^{18} \mathrm{~F}(\mathrm{p}, \alpha){ }^{15} \mathrm{O}$ astrophysical $S$-factor from the CNS-RIKEN experiment. The full dots are THM experimental data with the assumption of $J^{\pi}=3 / 2^{+}$for the resonance at $E=6460 \mathrm{keV}$ in ${ }^{19} \mathrm{Ne}$. The open ones corresponds to the assumption of $J^{\pi}=5 / 2^{-}$for the same level. Above $200 \mathrm{keV}$ data are not affected by the $J^{\pi}$ assumption for this level. The difference from the last assumption to the other possible value $1 / 2^{-}$and $3 / 2^{-}$is negligible within the errors. Refer to [20] for details.

physical interest, $A(x, C) D$ can be studied through a $A(B, C D)$ s process with three body in the final 
state. In order to do this, one has to select the events coming exclusively from the quasi-free reaction channel. In order to favour this channel, choosing a nucleus $B$ that has a strong $(x+s)$ cluster structure with low binding energy is of paramount importance. In this picture, particle $\mathrm{x}$ is the partecipant to the process while s is the spectator. A review of the method if reported in [18] mentioned above.

Typically THM measurement require a relatively simple detection setup. The situation becomes less favorable when one considers nuclear reactions initiated by unstable species. In the last two decades many radioactive ion beam (RIB) facilities were constructed but the intensities of the produced beams are much lower than those available when considering stable ones. In order to be able to measure the cross sections of reactions involving the use of these RIBs, even using indirect methods, high performance detection systems must be used, like the ASTRHO setup of LNS [19]. This detector array was used to measure the cross section of the ${ }^{18} \mathrm{~F}(\mathrm{p}, \alpha){ }^{15} \mathrm{O}$ process in Nova

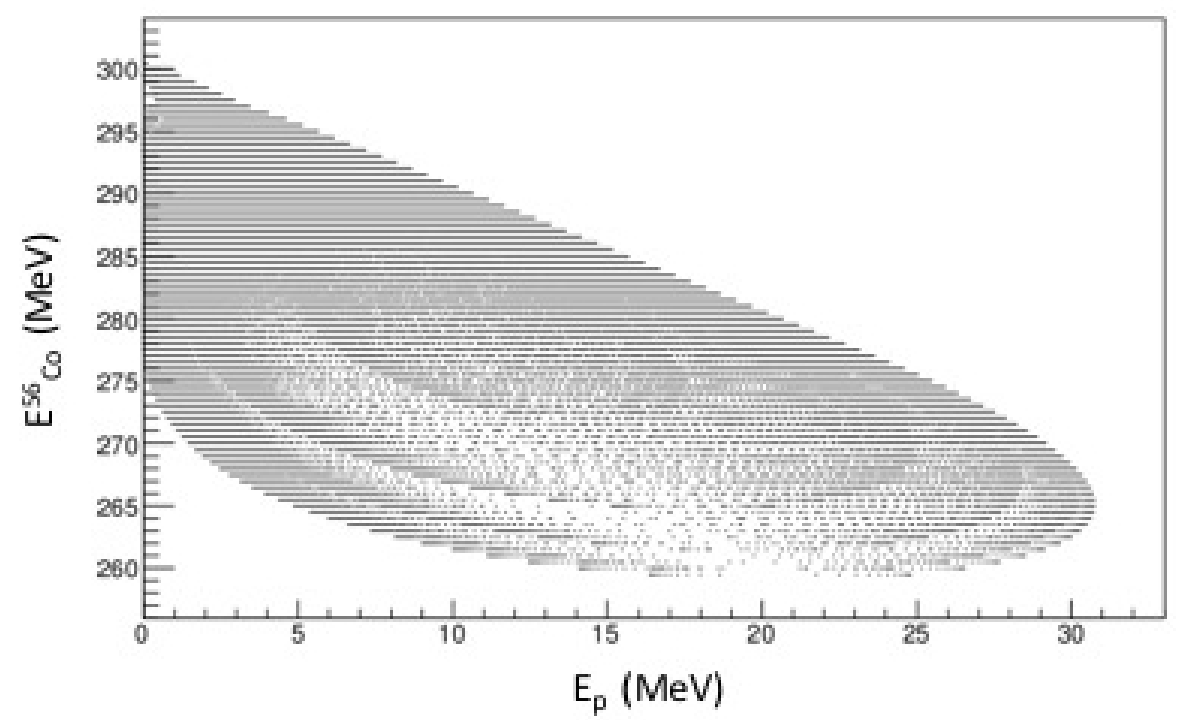

Figure 2: Kinematical calculation that show the energy-energy correlation between the outgoing particles in the three-body reaction $56 \mathrm{Ni}\left(\mathrm{d}, \mathrm{pp}_{s}\right) 56 \mathrm{Co}$ at $280 \mathrm{MeV}$ (total) $56 \mathrm{Ni}$ beam energy. Note that the outgoing angle of 56Co produced in the reaction is always lower than 1 degrees while the emitted proton spans an angular range $0-60$ degrees roughly.

conditions by applying the Trojan Horse Method to a reaction induced by a RIB, namely ${ }^{18} \mathrm{~F}(\mathrm{~d}, \alpha$ ${ }^{15} \mathrm{O}$ )n. The experiment was performed at the CRIB apparatus of Center for Nuclear Study (CNS) of The University of Tokyo, based at RIKEN campus in Wako, Japan. The results of this study have been reported in [20] and are shown as an example of the THM approach in figure 1. It has to be remarked that, in the same experiment, preliminary results have also been obtained for the neutron induced reaction ${ }^{18} \mathrm{~F}(\mathrm{n}, \alpha){ }^{15} \mathrm{~N}$. This shows that THM is an interesting tool for the measurement of neutron induced reaction, even when radioactive species are involved.

A key point in $\mathrm{SN}$ nucleosynthesis are neutron induced reactions on short lived radioactive species. The short mean life (d - ms) of many of the isotopes involved is a severe difficulty in the experimental study of these processes. With existing facilities, direct measurements are practically 
impossible. Among them the process $56 \mathrm{Ni}(\mathrm{n}, \mathrm{p}) 56 \mathrm{Co}$ is particularly important. We will measure its cross section by applying the THM to the $56 \mathrm{Ni}\left(\mathrm{d}, \mathrm{pp}_{s}\right) 56 \mathrm{Co}$.

To measure the cross section of the reaction $56 \mathrm{Ni}(\mathrm{n}, \mathrm{p}) 56 \mathrm{Co}$ via THM, a $56 \mathrm{Ni}$ beam with an energy of roughly $5 \mathrm{MeV} / \mathrm{A}$ has to be produced and a detection system allowing to measure the reaction products at very small angles ( $<1$ degrees) around the beam direction must be used. Indeed, it has to be stressed that in quasi-free conditions the spectator proton in $56 \mathrm{Ni}\left(\mathrm{d}_{,} \mathrm{pp}_{s}\right) 56 \mathrm{Co}$ (indicated by $\mathrm{p}_{s}$ ) basically stays in the target. So, to fully reconstruct the kinematics of the events, it is necessary to detect both the 56Co and the non-spectator proton produced in the reaction. A typical correlation spectrum between energy of the outgoing proton and of 56Co are shown in figure 2. Preliminary tests requiring short duration beam-time $(<=24 \mathrm{~h})$ will be performed both at CNS-RIKEN and LNS. The result of the measurement will finally be implemented in SN models.

\section{Acknoweledgments}

The author wishes to thank Prof. T. Kajino for many interesting and stimulating scientific discussions, for showing me the world of neutrinos in astrophysics and also for his strong encouragement in going down this track.

\section{References}

[1] G. Baur, Phys. Lett. B, 178, 135-138, (1986)

[2] C.Spitaleri. in Proceedings of the Fifth Hadronic Physics Winter Seminar, Folgaria - TN, Italy, Ed. World Scientific, Singapore, )(1990).

[3] S.Cherubini, V.N. Kondratyev, M.Lattuada, C.Spitaleri, D.Miljanic, M.Zadro, and G.Baur, ApJ, 457, $855,(1996)$.

[4] Letter of intent for KM3NeT 2.0, S. Adrián-Martínez et al., J. Phys. G: Nucl. Part. Phys. 43, (2016) 084001, doi:10.1088/0954-3899/43/8/084001

[5] C.E. Rolfs and W.S. Rodney, Cauldrons in the cosmos: Nuclear astrophysics, University of Chicago Press, 1988

[6] Letter of Intent:The Hyper-Kamiokande Experiment-Detector Design and Physics Potential, K. Abe et al., arXiv:1109.3262v1 [hep-ex]

[7] The GENIE Neutrino Monte Carlo Generator PHYSICS \& USER MANUAL (version 2.10.0), C. Andreopoulos et al., arXiv:1510.05494v1 [hep-ph]

[8] C. Spitaleri, L. Lamia, A. Tumino, RG Pizzone, S. Cherubini, A. Del Zoppo, P. Figuera, M. La Cognata, A. Musumarra, MG Pellegriti, A. Rinollo, C. Rolfs, S. Romano, S. Tudisco, Phys. Rev, C69, 055806, 2004.

[9] G.Baur and S.Typel. Prog. Theor. Phys. Suppl., 154, 333, 2004.

[10] A.M. Mukhamedzhanov, L.D. Blokhintsev, S. Brown, V. Burjan, S. Cherubini, V.Z. Goldberg, M. Gulino, B.F. Irgaziev, E. Johnson, K. Kemper, V. Kroha, M. La Cognata, L. Lamia, A. Momotyuk, R.G. Pizzone, B. Roeder, G. Rogachev, S. Romano, C. Spitaleri, R.E. Tribble, A. Tumino, Nucl. Phys., A787, 321C, 2007. 
[11] A. Tumino, C. Spitaleri, A. M. Mukhamedzhanov, G.G. Rapisarda, S. Cherubini, V. Crucillà, Z. Elekes, Z. Fulop, M. Gulino, G. Gyurky, G. Kiss, M. La Cognata, L. Lamia, F. Mudo, R.G. Pizzone, S. Romano, M.L. Sergi, E. Somorjai, Phys. Rev. Lett., 98, 252502 (2007).

[12] A. Tumino, C. Spitaleri, A.M. Mukhamedzhanov, G.G. Rapisarda, L. Campajola, S. Cherubini, V. Crucillà, Z. Elekes, Z. Fulop, L. Gialanella, M. Gulino, G. Gyurky, G. Kiss, M. La Cognata, L. Lamia, A. Ordine, R.G. Pizzone, S. Romano, M.L. Sergi, E. Somorjai, Phys. Rev., C 78, 064001, (2008).

[13] M. Gulino, C. Spitaleri, S. Cherubini, V. Crucillà, M. La Cognata, L. Lamia, R.G. Pizzone, S. Romano, M.L. Sergi, A. Tumino, Chengbo Li, Z. Elekes, E. Somorjai, V. Burjan, V. Kroha, A.M. Mukhamedzhanov, J. Phys. G: Nucl.Part.Phys. 37, 125105 (2010)

[14] M. La Cognata, A.M. Mukhamedzhanov, C. Spitaleri, I. Indelicato, M. Aliotta, V. Burjan, S. Cherubini, A. Coc, M. Gulino, Z. Hons, G.G. Kiss, V. Kroha, L. Lamia, J. Mrazek, S. Palmerini, S. Piskor, R.G. Pizzone, S.M.R. Puglia, G.G. Rapisarda, S. Romano, M.L. Sergi, A. Tumino, Apj., 739, L54, (2011).

[15] L. Lamia, C. Spitaleri, V. Burjan, N. Carlin, S. Cherubini, V. Crucillà, M.G. Munhoz,M.G. Del Santo, M. Gulino, Z. Hons, G.G. Kiss, V. Kroha, S. Kubono, M. La Cognata, Chengbo Li, J. Mrazek, A.M. Mukhamedzhanov, R.G. Pizzone, S.M.R. Puglia, Q. Wen, G.G. Rapisarda, C. Rolfs, S. Romano, M.L. Sergi, E. Somorjai, F.A. Souza, A.S. de Toledo, G. Tabacaru, A. Tumino, Y. Wakabayashi, H. Yamaguchi, S.H. Zhou J. Phys. G: Nucl.Part.Phys., 39, 015106 (2012)

[16] M. Gulino, S. Cherubini, G.G. Rapisarda, S. Kubono, L. Lamia, M. La Cognata, H. Yamaguchi, S. Hayakawa, Y. Wakabayashi, N. Iwasa, S. Kato, T. Komatsubara, T. Teranishi, A. Coc, N. De Sereville, F. Hammache, C. Spitaleri, J. Phys. Conf. Ser., 420012149 (2013)

[17] M. Gulino, C. Spitaleri, X.D. Tang, G.L. Guardo, L. Lamia, S. Cherubini, B. Bucher, V. Burjan, M. Couder, P. Davies, R. deBoer, X. Fang, V.Z. Goldberg, Z. Hons, V. Kroha, L. Lamm, M. La Cognata, Chengbo Li, C. Ma, J. Mrazek, A.M. Mukhamedzhanov, M. Notani, S. O’Brien, R.G. Pizzone, G.G. Rapisarda, D. Roberson, M.L. Sergi, W. Tan, I.J. Thompson, M. Wiescher, Phys.Rev., C 87, 012801 (2013)

[18] R.E. Tribble, C.A. Bertulani, M. La Cognata, A.M. Mukhamedzhanov and C. Spitaleri, Rep. Prog. Phys. 77, 106901 (2014)

[19] S. Cherubini et al., ASTRHO: an Array of Silicons for TRojan HOrse studies with RIBs. In preparation

[20] S. Cherubini, M. Gulino, C. Spitaleri, G.G. Rapisarda, M. La Cognata, L. Lamia, R.G. Pizzone, S. Romano, S. Kubono, H. Yamaguchi, S. Hayakawa, Y. Wakabayashi, N. Iwasa, S. Kato, T. Komatsubara, T. Teranishi, A. Coc, N. de SÃl'rÃl'ville, F. Hammache, G. Kiss, S. Bishop, and D. N. Binh, Phys. Rev. C 92, 015805 (2015)

[21] M. Fukugita -T. Yanagida, Physics of Neutrinos and Application to Astrophysics, Springer-Verlag Berlin Heidelberg, 2003 